\title{
Diseño de una oficina de gestión de proyectos para la empresa Instelmec SAS
}

\author{
Design of a project management office for the company Instelmec SAS
}

Fecha de entrega: 28 de mayo 2019

Fecha de aceptación: 27 de febrero 2020

\section{Leandro Reyes, Michelle Briggite Quiroga y Yon Jairo Trujillo}

Escuela de Administración de Negocios, Universidad EAN, Calle 79 No. 11 - 45, Bogotá D.C., Colombia,

1reyesjo936@universidadean.edu.co,mquiroga8136@universidadean.edu.co,ytrujill5420@universidadean.edu.co

El presente trabajo tiene como objetivo generar la propuesta para la implementación de una Oficina de Gestión de Proyectos OGP para la compañia Instelmec SAS. Esto permite alinear los procesos que se llevan a cabo actualmente en los proyectos con los objetivos estratégicos propuestos por la organización. Actualmente los proyectos se ejecutan en base a la experiencia del personal. La organización no cuenta con procesos estandarizados que permitan ejecutar los proyectos de una manera organizada. Con base a la revisión de los acervos de la gestión de proyectos referenciados en el presente estudio, se aplican al análisis de la información de Instelmec para generar un plan de implementación de la OGP. Se definen sus objetivos, alcance, limitaciones, misión, visión, roles y demás aspectos relacionados con los proyectos de Instelmec SAS. En el desarrollo de esta investigación se aplicaron diferentes instrumentos de medición, como son encuestas, entrevistas, auditorias y modelo de madurez, para determinar en la organización la OGP más adecuada. Se analizó la organización desde diferentes puntos de vista, como es la gestión de portafolio de proyectos, cultura organizacional y efectividad en proyectos, las prácticas de gestión de proyectos y la medición de madurez en gestión de proyectos. Con los resultados obtenidos se diseñó una OGP básica.

Palabras clave: oficina de gestión de proyectos, efectividad, cultura organizacional, madurez
The present work aims to generate the proposal for the implementation of a Project Management Office for the company Instelmec SAS. This allows to align to the processes currently carried out with the strategic objectives proposed by the organization. Projects are currently executed based on the staff experience. Under the above considerations, the organization does not have standardized processes that allow projects to be executed in an organized manner. Based on the review of the project management assets referenced in this study, they are combined and applied to the analysis of the information collected in the Instelmec organization to subsequently generate an implementation plan for the Project Management Office and on which its objectives, scope, limitations, mission, vision, roles and other aspects related to Instelmec SAS projects are defined. In the development of this research, different measurement instruments were applied, such as surveys, interviews, audits and maturity model, in order to determine the most appropriate Project Management Office in the organization. The organization was analyzed from different points of view, such as project portfolio management, organizational culture and project effectiveness, project management practices and project management maturity measurement. With the results obtained, a basic Project Management Office was designed.

Keywords: project management office, effectiveness, organizational culture, maturity

\section{Introducción}

Este trabajo se enmarca en el contexto de aplicación empresarial, el cual se basa en el diseño de una Oficina de Gestión de Proyectos OGP para la empresa Instelmec S.A.S., empresa que trabaja en el sector de diseño, construcción y mantenimiento de obras civiles, arquitectura y urbanismo. El enfoque del presente trabajo consiste en dar respuesta a la pregunta ¿cómo se puede implementar una unidad organizacional que centralice la gestión de proyectos en la organización Instelmec SAS?. El objetivo general es diseñar una OGP para la empresa Instelmec SAS con lo que se pretende dar una solución a la problemática que hay actualmente al interior de la compañía en el manejo y gestión de sus proyectos. Para 
el desarrollo de la investigación se utilizaron diferentes herramientas de investigación como son la entrevista, encuestas y auditorias, cuyos resultados fueron analizados para determinar el estado actual de la empresa en temas de gestión de proyectos y proponer lineamientos que permitan llevarla al crecimiento y posicionamiento en nuevos mercados, incrementando sus utilidades y haciéndola más sólida económicamente. Se debe indicar que este análisis es independiente del nivel de producción de la empresa. Con los resultados obtenidos se propone una OGP básica que se adapta a las condiciones actuales de Instelmec, acompañado de un plan de implementación, el cual incluye diferentes aspectos como son las fases de implementación, métricas de calidad, análisis de riesgos y presupuesto para llevar a cabo el proyecto.

\section{Oficina de Gestión de Proyectos OGP}

Para la gestión de proyectos no es suficiente tener profesionales y una metodología enfocada en la estrategia organizacional, también es importante tener una gobernabilidad de los proyectos, una dirección apropiada, utilizar metodologías con estándares, herramientas, seguimiento y control, de acuerdo con las necesidades del cliente y es esto precisamente las funciones de una OGP. La OGP se define como un marco en el que la dirección de portafolios, de programas y de proyectos integradas con los elementos facilitadores de la organización a fin de alcanzar los objetivos estratégicos (PMI, 2017). Jiménez et al. (2019) clasifica las OGPs de acuerdo con el desarrollo alcanzado por la OGP. Por otro lado, PMI (2017) detalla los diferentes roles, funciones y tipos de OGP y los roles de los directores de proyectos.

La cultura, efectividad y madurez en el ámbito organizacional son factores que influyen y son determinantes en el desarrollo de los proyectos. Muchas veces se ven favorecidos o perjudicados los proyectos por este componente que incluso puede cambiar entre equipos o áreas de la misma organización (Ariza, 2017a, 2017b; Gray y Larson, 2009; Jiménez et al., 2019; Hill, 2008; PMI, 2017).

La empresa Instelmec tiene más de 20 años de trayectoria en la consultoría en proyectos de arquitectura e ingeniería (Global Group Col SAS). No tiene metodologías ni procesos para la ejecución de los proyectos, las actividades se realizan de manera informal y empírica desarrollada con base a la experiencia y capacidad de dirección de los involucrados, adicionalmente en los proyectos no está asociada la figura de un líder específico, por el contrario, varias personas según su disponibilidad atienden y se les hacen responsables según los requerimientos internos y externos de los proyectos (Moreno, D. comunicación personal, 11 de agosto de 2018). Instelmec es una organización funcional enfocada en la ejecución de proyectos, por tal razón la cantidad de empleados (directos o indirectos) varía constantemente al corte de cada año dado que los proyectos se liquidan y los empleados se reducen.

Esta investigación fue realizada con apoyo de la Gerencia de Instelmec durante un periodo de tiempo de diez meses, iniciando en agosto 2018 y finalizando en mayo 2019. Los stakeholders fueron los empleados de la empresa Instelmec, cuyo rol de participación en los proyectos fue principalmente el de integrantes del equipo del proyecto $(60 \%)$, con una permanencia en la organización de 3 a 5 años (48\%) y su rango de edad era menor a 30 años (72\%). Se utilizaron diferentes técnicas para la recolección de datos evaluando cuatro (4) aspectos principales: La gestión del portafolio de proyectos con la entrevista, la cultura organizacional y la efectividad de los proyectos con encuesta, las prácticas de gestión de proyectos con la auditoría y la madurez en gerencia de proyectos aplicando el cuestionario de modelo de madurez (Kerzner, 2002). El instrumento que se empleó contiene entrevistas y encuestas, que fueron diseñadas por Ariza (2017a).

\section{Gestión del portafolio de proyectos}

Para la medición de la gestión de portafolio se utilizó la entrevista del tipo semiestructurada diseñada por Ariza (2017a). La herramienta se encuentra dividida en dos secciones, la primera relacionada con las prácticas de gestión del portafolio de proyectos y la segunda relacionada con la cultura y efectividad organizacional asociada a la gestión del portafolio de proyectos (Ariza, 2017b). Considera preguntas estratégicas que permiten obtener información alineada al tipo de investigación planteada, permitiendo conocer el estado actual de la empresa, información base para definir el tipo de oficina de proyectos diseñada para la empresa en estudio. En la realización de las entrevistas se seleccionó una muestra no probabilística con empleados de la empresa Instelmec que se encontraban trabajando en la división de proyectos. 
Las entrevistas se realizaron de manera presencial en las instalaciones de la empresa del 15 y 22 de febrero de 2019, entrevistando a tres personas relacionadas con la ejecución de proyectos de las diferentes áreas como son: el coordinador de proyectos, el profesional responsable de calidad y un líder de proyectos, con el fin de contar con diferentes puntos de vista sobre la manera como ven los funcionarios la aplicación de la gestión de proyectos en la organización.

\section{Cultura organizacional y efectividad en el ámbito de los proyectos}

La medición de la cultura organizacional y efectividad se realizó por medio de una encuesta (Ariza, 2017a), cuyo medio de uso fue correo electrónico, con el objetivo de recolectar información requerida para evaluar aspectos culturales de los stakeholders, evaluando los 32 indicadores de cultura organizacional en el ámbito de los proyectos y 11 indicadores de efectividad de los proyectos. El instrumento utilizado de encuesta contiene preguntas para medir la percepción que tienen los empleados en el ámbito de la ejecución de proyectos agrupadas en 4 dimensiones: Gobernabilidad cultural de proyectos, aprendizaje en el ámbito de proyectos, integración organizacional y relacionamiento entre stakeholders. La información resultante permite identificar aspectos de la cultura organizacional que afecta la gestión de proyectos, la cual fue de utilidad en el diseño de la oficina de proyectos adecuada para la compañía. En términos de efectividad se relaciona con el éxito y calidad, bajo los términos de la ejecución de un proyecto en una organización, Ariza (2017a) describe tres factores para evaluar la efectividad de la ejecución de proyectos, se incluyen criterios de soporte a nivel ejecutivo, disciplina y resultados en los proyectos, después hace un constructo epistemológico para confrontar la teoría con la evaluación bajo diferentes perspectivas de los stakeholders.

La encuesta creada por Ariza (2017a) permite identificar los criterios de efectividad de la organización en la ejecución de proyectos, las cuales se agrupan en 3 dimensiones que son: cumplimiento de expectativas de los stakeholders, satisfacción del equipo y logro de compromisos. Con la información recolectada se obtiene la relación que hay entre los aspectos culturales de la compañía versus la percepción de efectividad que tiene los empleados en la gestión de los proyectos, permitiendo relacionar aspectos que orienten al diseño de la OGP para la empresa.

\section{Prácticas de gestión de proyectos}

De acuerdo con los proyectos ejecutados en Instelmec en el año 2018, se realizó una auditoria en cada una de las fases de los proyectos, con el objetivo de identificar la correspondencia de aplicación de prácticas de gestión de proyectos. Como la empresa es una Mipyme del sector de diseños de obras civiles, la encuesta fue realizada a un grupo de 4 personas del área de diseños, que conforman el $65 \%$ de la planta de personal de la empresa perteneciente a esta área. Se escogieron dos proyectos que fueron auditados utilizando una lista de chequeo que contiene preguntas de prácticas de gestión de proyectos según el ciclo administrativo de un proyecto propuesto por PMI (2017) discriminadas por grupos de proceso y áreas de conocimiento. Esta lista de chequeo permitió identificar la visión que tiene Instelmec en la administración de los proyectos durante todas las etapas de ejecución, lo que encaminará a proponer buenas prácticas en este ámbito dentro de la OGP, que conlleve a la realización de los proyectos con éxito.

\section{Medición de la madurez en gerencia de proyectos}

Teniendo en cuenta que la empresa no cuenta con procedimientos formales para la gestión de proyectos, se tomó como guía la definición de madurez de proyectos para evaluar el nivel 1, con el fin de establecer si tienen un nivel básico de conocimientos en proyectos que permita superar este nivel bajo los riesgos que la organización esté dispuesta a tolerar. El cuestionario evalúa la aplicación y conocimiento de los principios fundamentales de la gestión de proyectos y su terminología asociada. Los requisitos para completar el nivel 1 se pueden cumplir a través de una buena comprensión de la guía del PMBOK тм preparada por el PMI (Kerzner, 2002). Las 80 preguntas cubren el PMBOK y los principios básicos de la gestión de proyectos. Hay cinco respuestas para cada pregunta (A, B, C, D y E) con única respuesta. Se evaluaron 8 categorías basadas en el PMBOK que son: alcance/gestión de la integración, tiempo, costos, recursos humanos, adquisiciones, calidad, riesgos y comunicación (Kerzner, 2002). 
La calificación es de 0 para las respuestas incorrectas y 10 para las correctas de acuerdo con lo propuesto por Kerzner (2002), si la puntuación sumada es 60 o más puntos para cada una de las ocho categorías, entonces la organización tiene un conocimiento razonable de los principios básicos de la gestión de proyectos. Si la puntuación es de 60 o más en todas menos una o dos de las categorías, la organización posee todo el conocimiento que necesita de los principios básicos de gestión de proyectos. Si el puntaje es menor a 60 en cualquier categoría, existe una deficiencia. Para puntajes de menos de 30 en cualquier categoría, parecen necesarios programas rigurosos de capacitación sobre principios básicos, la organización parece altamente inmadura en la gestión de proyectos (Kerzner, 2002). Este análisis fue realizado con base al diagnóstico previo de los puntos anteriores, su experiencia y formación en gerencia de proyectos.

\section{Resultados del estudio}

La toma de información de campo se realizó los días 15 y 22 de febrero de 2019, en las instalaciones de la empresa, encuestándose los 25 empleados, obteniéndose los siguientes resultados para cada una de las mediciones realizadas.

\section{Prácticas de gestión del portafolio de proyectos}

Se encontró acuerdo en el $100 \%$ de los entrevistados, en que en la empresa no existe una definición de portafolio de proyectos, solo tienen el concepto de proyectos que se gestionan de manera independiente y cuya información se registra a través de los ingenieros encargados de liderarlos. Se realizan proyectos en el sector de recreación, educación, salud y vivienda. El Jefe Funcional consolida la información dada por los líderes de proyectos y la presenta al Director y a la Junta Directiva.

En cuanto a la gestión de riesgos, no se tiene evidencia de la existencia de una metodología para la identificación, análisis y monitoreo de riesgos, por lo tanto, la gestión que se realiza en la ejecución de proyectos es mínima. En ocasiones los directores de proyecto realizan reuniones con los interesados y equipo del proyecto para realizar la identificación de los riesgos de cada uno de los proyectos que tienen a su cargo, pero una vez que se identifican no se realiza ninguna gestión ni proceso adicional. La organización es consciente de la existencia de los riesgos y para cubrirse destina un porcentaje a imprevistos. El porcentaje de imprevistos es a criterio del profesional encargado o del Gerente General. Los riesgos se determinan por cada proyecto de manera informal y no están asociados al portafolio de proyectos. Algunos ejemplos de riesgos afectan el cumplimiento en la construcción de las obras.

Se encontró acuerdo en el $100 \%$ de los entrevistados en que los proyectos que serán desarrollados por Instelmec los determina el Gerente General quien realiza la consecución de estos a través de sus conexiones. Lo que se observó con el resultado de las encuestas es que el Gerente General es quien lidera la empresa y toma de decisiones en la realización de un proyecto. El Gerente General es el único que se encuentra empoderado para cambiar el estado de un proyecto que esté en fase de ejecución. Los cambios que se realizan a los proyectos se documentan en un formato de control de cambios que se tiene elaborado para este fin. Cuando se realiza un cambio se da la información al director de proyecto. Los criterios para cancelar o suspender un proyecto que ya está siendo desarrollado, incluyen las demoras o la no aprobación de las licencias ambientales requeridas para su construcción. Otro factor es el sobrecosto dado que estos se ejecutan a todo costo. Se realiza el análisis financiero y si este no es llamativo el proyecto se suspende o cancela. El criterio para determinar si un proyecto es rentable o no está en cabeza del Gerente General. El porcentaje de utilidad en los proyectos es información confidencial de la empresa.

En cuanto a la definición de recursos sí hay discrepancia en los resultados de la entrevista puesto que cada persona da un concepto diferente arrojando esto que los jefes de área, el gerente comercial o el Gerente General son los que realizan esta asignación. Para el caso de disponibilidad de personal para los proyectos, las respuestas arrojaron que la empresa no cuenta con un software o herramienta de apoyo para llevar el control de la ocupación del personal. Por lo tanto, este control se lleva de manera empírica a criterio del jefe. Las personas que laboran en los proyectos no tienen dedicación exclusiva, trabajando de forma paralela en otros proyectos. En cuanto a cultura organizacional asociada a la gestión del portafolio de proyectos, las personas entrevistadas manifestaron que en la empresa se incentiva una cultura de participación en las reuniones, donde las personas tienen libertad de decir el estado real 
de los proyectos sin miedo a ser atacadas negativamente. Se encontró un $100 \%$ de coincidencia en que el principal problema que enfrenta la gestión del portafolio de proyectos es la falta de continuidad del personal y la concentración de la mayoría de las decisiones en el Gerente General.

\section{Valoración de la cultura y efectividad de los proyectos}

Los resultados de la encuesta de cultura organizacional y efectividad en el ámbito de los proyectos fueron tomados por las respuestas dadas por 25 empleados y se muestran en la Tabla 1.

Tabla 1: Resultados de la encuesta de cultura organizacional en el ámbito de proyectos

\begin{tabular}{|l|c|}
\hline Variable & Puntaje \\
\hline Alineación estratégica de proyectos & 7.23 \\
\hline Entendimiento del ámbito de los proyectos & 7.54 \\
\hline Adopción de prácticas de gestión de proyectos & 7.12 \\
\hline $\begin{array}{l}\text { Valoración del conocimiento asociado a los } \\
\text { proyectos }\end{array}$ & 7.16 \\
\hline Cohesión entre los stakeholders & 7.01 \\
\hline Empoderamiento & 7.32 \\
\hline Consideración de los stakeholders & 7.31 \\
\hline Adaptación para la solución de problemas & 7.14 \\
\hline Puntaje final Cultura & 7.23 \\
\hline
\end{tabular}

Todos los resultados de los indicadores de cultura organizacional se encuentran por encima de 7 puntos estando cada uno muy cercano al otro en un rango entre 1 y 10. Esto muestra que la percepción de los empleados con respecto a las características de la cultura organizacional es similar y que hay posibilidades de mejora en todos los aspectos culturales evaluados.

Al observar los resultados de las respuestas de los encuestados en la Figura 1, se ve una percepción más desfavorable en los componentes que corresponden a cohesión entre los stakeholders, específicamente en la característica de cultura relacionada con la existencia de una perspectiva común entre diferentes áreas para el desarrollo de los proyectos, de acuerdo a Ariza (2017a). Este puede ser un aspecto importante para promover la oficina de proyectos. Los resultados para los indicadores de efectividad en el ámbito de los proyectos se muestran en la Figura 2 y en la Tabla 2.

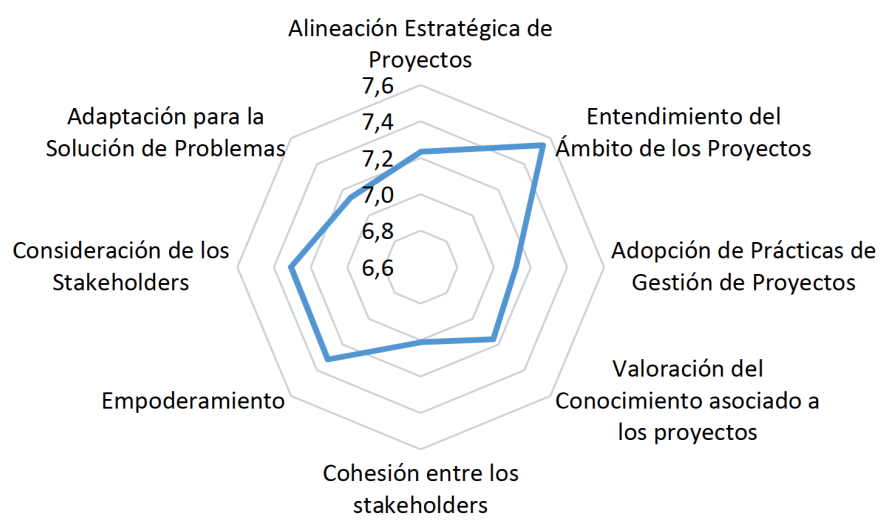

Figura 1: Resultados en cultura organizacional

Tabla 2: Resultados encuesta efectividad en el ámbito de proyectos

\begin{tabular}{|l|c|}
\hline Variable & Puntaje \\
\hline 1. Cumplimiento de expectativas & 7.76 \\
\hline 2. Satisfacción del equipo de proyecto & 7.28 \\
\hline 3. Logro de compromisos & 7.28 \\
\hline Puntaje final Efectividad & 7.44 \\
\hline
\end{tabular}

Sin dejar de lado los resultados de la percepción que tienen los empleados de la efectividad de los proyectos en Instelmec (Figura 2), se observa una mejor percepción en los componentes de cumplimiento de expectativas, como en un análisis de variables y su correlación se podrá aplicar modelos que relacionan directamente estos dos aspectos como un factor determinante para el éxito de proyectos y por tanto información valiosa en el momento de decidir cuál oficina de proyectos es la más adecuada, la dirección que debería tomar y la estrategia que puede adaptar para el camino de fortalecer la organización en la vía de estandarización de metodologías en proyectos.

La similitud de las valoraciones tanto en las categorías culturales como en los indicadores de efectividad, hacen pensar que se presentó un posible sesgo relacionado con el deseo de obtener aprobación social por parte de la persona que realiza la valoración (Podsakoff et al., 2003). Se trató de disminuir la presentación de este posible sesgo, indicándole a los participantes que su información era confidencial y que solo serían públicos los resultados estadísticos, los cuales serían utilizados exclusivamente para fines académicos. Adicionalmente, teniendo en cuenta que los valores descriptivos de la media y mediana 
son muy cercanos, se puede concluir que la distribución normal es simétrica.

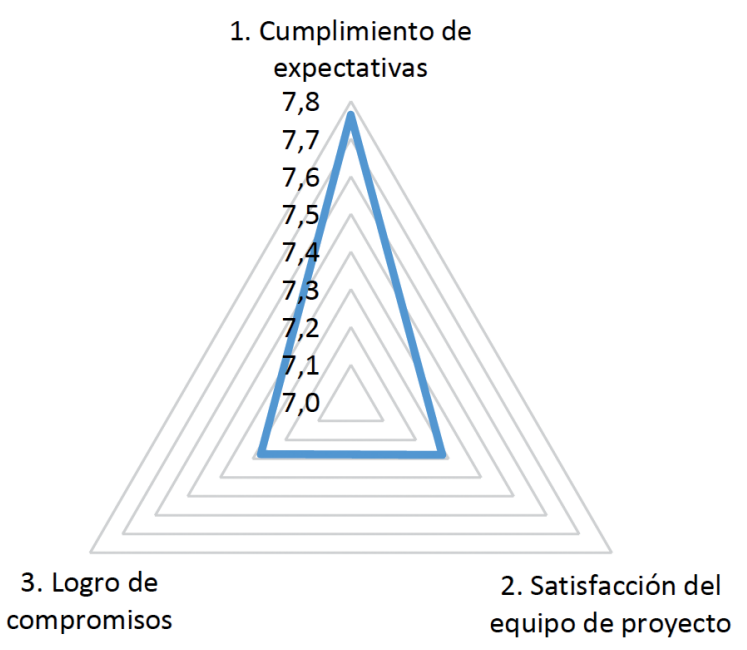

Figura 2: Resultados de efectividad

\section{Prácticas de gestión de los proyectos}

Considerando que la empresa en estudio no cuenta con un sistema de gestión de proyectos implementado, se realizó una encuesta en las instalaciones de la empresa el día 15 de febrero de 2019, para identificar las prácticas que la empresa utiliza para la gestión de dos proyectos, los cuales se encuentran en fase de ejecución. De las auditorías se analizaron los resultados por cada área de conocimiento del PMI, los cuales se muestran en la Tabla 3.

Tabla 3: Resultados prácticas de gestión de proyectos

\begin{tabular}{|l|c|}
\hline Área de conocimiento & Desempeño, \% \\
\hline Alcance & 50 \\
\hline Stakeholders & 67 \\
\hline Tiempo & 0 \\
\hline Costo & 0 \\
\hline Recursos humanos & 67 \\
\hline Comunicaciones & 0 \\
\hline Calidad & 0 \\
\hline Riesgos & 0 \\
\hline Adquisiciones y contratación & 33 \\
\hline Cambio & 33 \\
\hline
\end{tabular}

En la Figura 3 se presentan los resultados por grupos de procesos de la encuesta realizada.

Con base a estos resultados se determina que la compañía se preocupa al comienzo de los proyectos de realizar las adquisiciones de recursos y contratación de personal que se

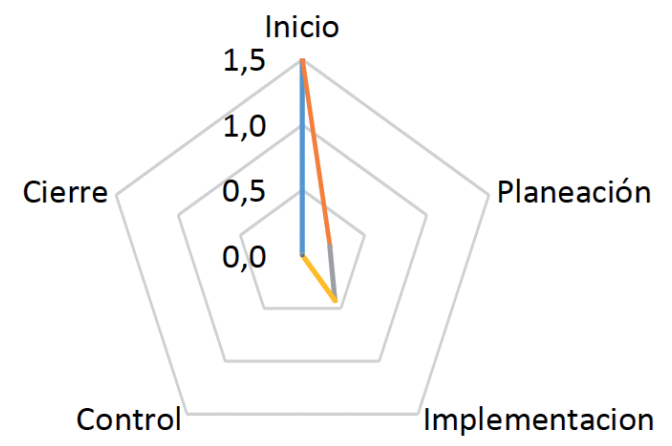

Figura 3: Vista por grupo de procesos en Instelmec

requiera, sin embargo, no verifica la experiencia del personal contratado ni realiza seguimiento al cumplimiento de los compromisos que los contratistas adquieren. En cuanto a la gestión de stakeholders la empresa escucha y toma nota de los requerimientos de los clientes particularmente y define los objetivos con base en las necesidades de estos. Pero no cuantifica el trabajo necesario para cumplir con los objetivos ni define los entregables del proyecto. No existe ninguna práctica que le permita a la empresa definir y hacer seguimiento a las medidas de calidad, por lo cual no existe ninguna metodología que permita asegurar la aceptación de los entregables por parte del cliente. Los cambios que se presentan en los proyectos con frecuencia son aprobados, considerando que no existe un mecanismo que le permita a Instelmec analizar y tomar decisiones para aceptar o negar un cambio solicitado por el cliente. No existe un plan de comunicaciones que permita a la compañía mantener información actualizada del estado del proyecto y por consiguiente no es posible generar registros de lecciones aprendidas ni reportes que permitan realizar presentaciones a los clientes. No se generan cronogramas aprobados por el cliente, por lo tanto, no es posible reportar actividades de avances y detectar desviaciones que permitan generar alertas para tomar acciones correctivas. Se asignan roles y se generan responsabilidades en el equipo de proyectos, sin embargo, no se monitorea el desempeño del equipo de proyectos a lo largo de la ejecución de estos.

Instelmec no relaciona los riesgos asociados a los proyectos y por tanto no realiza ninguna gestión asociada a mitigaciones. Los resultados obtenidos de prácticas de gestión de proyectos para cada área de conocimiento muestran que Instelmec tiene un valor bajo de cumplimiento notándose la carencia de una metodología de gerencia de proyectos. La gestión de stakeholders y recursos humanos 
son los que mejor resultados obtuvieron en la empresa con un $67 \%$ y la de menor puntuación fue la gestión de la calidad, comunicaciones, costos y tiempo con un valor de $0 \%$, haciendo imprescindible y necesario la centralización de la gestión de los proyectos a través de un área que sea responsable del seguimiento a las prácticas de gestión de proyectos y su mejora continua.

\section{Encuesta de madurez}

Se realizó la evaluación de madurez de nivel 1 de Kerzner (2002) obteniendo el resultado por cada pregunta, donde al tener la respuesta de acuerdo con la calificación asignada por Kerzner, se le asignaba 10 y si la respuesta no estaba de acuerdo se le asignaba 0 (Tabla 4). Este instrumento de evaluación se puede utilizar para medir el conocimiento de una persona o el conocimiento de una organización (Kerzner, 2002).

Tabla 4: Resultado puntos por categoría

\begin{tabular}{|c|c|}
\hline Categorías & Total \\
\hline Alcance/Gestión de la integración & 50 \\
\hline Gestión del tiempo & 20 \\
\hline Manejo de costos & 10 \\
\hline Gestión de recursos humanos & 30 \\
\hline Dirección de procuración & 30 \\
\hline Gestión de la calidad & 40 \\
\hline Gestión de riesgos & 30 \\
\hline Gestión de la comunicación & 50 \\
\hline Puntaje total & 260 \\
\hline
\end{tabular}

La evaluación de nivel 1 de la empresa dio como resultado 260 puntos, las categorías de alcance/gestión de integración y gestión de comunicación son las categorías con mayor puntuación, sin embargo, no superaron los 60 puntos, en la categoría de manejo de costos se tuvo la menor calificación, por lo que se recomienda hacer un refuerzo importante en todas las categorías de gestión de proyectos, especialmente fortalecer ámbitos de comunicación entre los stakeholders.

\section{Propuesta de diseño de la OGP}

Para la compañía Instelmec se diseñó una oficina de proyectos básica cuyo procedimiento contempló la elaboración de su misión, visión, objetivos estratégicos, roles, funciones y responsabilidades y organigrama con la ubicación en la compañía. El periodo propuesto para la implementación de la OGP fue de un año y tendrá a cargo las siguientes actividades:

- Entrenar el equipo de trabajo que conforma la OGP para fortalecer su conocimiento y que sea aplicado en la ejecución de la labor.

- Difundir el plan de comunicaciones y realizar una campaña de publicidad en la que se socialice a todos los empleados de la compañía, la implementación de la OGP y los beneficios que esta conllevará a la organización.

- Conformar y listar las personas que conforman los equipos de trabajo para los proyectos.

- Desarrollar sesiones de trabajo con los equipos de proyecto para darles a conocer la metodología de gestión de proyectos que debe ser aplicada en la ejecución de estos.

- Definir las personas que conformaran los equipos de trabajo de los nuevos proyectos que la empresa realice.

- Realizar auditorías a los proyectos para verificar la aplicabilidad de la metodología de proyectos, levantando las acciones de mejora o no conformidades buscando siempre la mejora continua (Montes et al., 2018).

- Desarrollar nuevas capacitaciones y reentrenamientos a las personas o equipos de proyecto que lo requieran, con el fin de incrementar su know how para que sea empleado en los proyectos.

- Monitorear y gestionar los riesgos de los proyectos con el fin de mitigarlos para que en lo posible no lleguen a su materialización.

- Llevar un control sobre los proyectos actuales y listar los nuevos proyectos para realizar el seguimiento.

- Asesorar a los gerentes de proyecto en la toma de decisiones cuando sea requerido.

- Monitorear y controlar los cambios que surjan en la ejecución de los proyectos.

- Comunicar las nuevas normas y ajustes a las metodologías que se lleguen a dar para la gestión de los proyectos.

- Seguimiento continuo a la ejecución de los proyectos y apoyo en la estructuración de nuevos proyectos. 


\section{Conclusiones}

Se realizó una investigación del tipo mixto dado que tuvo un enfoque tanto cualitativo como cuantitativo. Para la toma de información se realizaron encuestas a 25 empleados, entrevistas a 3 colaboradores que trabajan en proyectos, auditorías a 2 proyectos, cuyo objetivo determinó el conocimiento y nivel de gestión de proyectos que existe en la empresa y a partir del análisis de los resultados proponer una Oficina de Gestión de Proyectos OGP apropiada para la organización. La respuesta a la pregunta ¿cómo se puede implementar una unidad organizacional que centralice la gestión de proyectos en la organización Instelmec?, se tiene que, bajo el resultado arrojado de las entrevistas y las encuestas, es necesario centralizar la gestión de proyectos a través de una OGP básica, compuesta por un Gerente, un ingeniero y un apoyo, que podrá ir incrementando su personal dependiendo del tamaño y la cantidad de los proyectos. Para cumplir el objetivo de diseñar una OGP, el trabajo de investigación propone la implementación de una OGP cuyo objetivo es gestionar los proyectos a través de una metodología difundida a todos los interesados bajo un plan de comunicaciones. Al personal se capacitará y entrenará para la ejecución de los proyectos.

En cuanto al diagnóstico de prácticas de gestión de proyectos en la organización, el resultado del estudio arrojó que los proyectos actualmente son ejecutados empíricamente con base a la experiencia obtenida por los gerentes de proyectos, es decir, la empresa no cuenta con una metodología de proyectos. Para el desarrollo de una estructura organizacional y de la OGP apropiada para la organización, del análisis de resultados se encontró que la empresa está categorizada en nivel 1 de madurez y por lo tanto se estructuró la implementación de una OGP básica. Para el desarrollo del plan de implementación de la OGP se contempló una serie de actividades en las cuales se destaca la elaboración de una metodología, un plan de capacitaciones y de comunicaciones, con el fin que la organización cuente con una oficina que estructure, soporte y apoye en la ejecución de los proyectos.

Se propone como próximas investigaciones elaborar una metodología híbrida de proyectos que aplique a este tipo de empresas pequeñas utilizando como base los diferentes estándares tradicionales como las metodologías ágiles, lo cual sería de gran funcionalidad dado que ninguna metodología es completa y se podrían utilizar las buenas prácticas de cada una y elaborar una metodología que aplique a la gestión de proyectos de acuerdo con las especialidades de las empresas. También se propone estudiar el efecto de la OGP en la producción anual de la empresa.

\section{Agradecimientos}

Se agradece a la Dra. Dora Alba Ariza Aguilera por compartir su tiempo, brindar sus conocimientos y asesoría durante toda esta investigación. A la empresa Instelmec SAS por permitir visitar sus instalaciones, facilitar el tiempo y brindar la información requerida para realizar este trabajo de investigación.

\section{Referencias}

Ariza, D.A. (2017a). Construcción de un modelo de cultura organizacional en el ámbito de los proyectos y aplicación al sector de tecnología de la información en Colombia. Tesis doctoral, Universidad EAN, Bogotá, Colombia

Ariza, D.A. (2017b). Efectividad de la gestión de los proyectos: una perspectiva constructivista. Obras y Proyectos 22, 75-85

Gray, C.F. y Larson, E.W. (2009). Administración de proyectos. Mc Graw Hill, Mexico D.F.

Hill, G.M. (2008). The complete project management office handbook. Auerbach Publications- Taylor \& Francis Group.

Jiménez, J.F., León, D.A., Mahecha, J.S., Manco, J.M. and Pita, M.G. (2019). Diseño y plan de implementación para el montaje de una Oficina de Gestión de Proyectos (OGP) en MYV Consultores Asociados SA. Obras y Proyectos 26, 51-64

Kerzner, H. (2002). Strategic planning for project management using a project management maturity model. John Wiley y Sons, New York, USA

Montes, F.J., Paredes, L., y Alarcón, A. (2018). Cultura organizacional y grado de madurez en gestión de proyectos aplicación entidad sin ánimo de lucro en Bogotá "Corporación Fomentar Desarrollo”. Trabajo de título, Universidad Católica de Colombia

PMI (2017). Guía de los fundamentos para la dirección de proyectos (Guía del PMBOK). Project Management Institute, Inc. Pennsylvania, USA

Podsakoff, P.M., MacKenzie, S.B., Lee, J.Y. and Podsakoff, N.P. (2003). Common method biases in behavioral research: A critical review of the literature and recommended remedies. Journal of Applied Psychology 88(5), 879-903 